\title{
Arrhythmic Pulses Detection Using Lempel-Ziv Complexity Analysis
}

\author{
Lisheng $\mathrm{Xu},{ }^{1}$ David Zhang, ${ }^{2}$ Kuanquan Wang, ${ }^{1}$ and Lu Wang ${ }^{1}$ \\ ${ }^{1}$ Department of Computer Science and Engineering, School of Computer Sciences and Technology, \\ Harbin Institute of Technology (HIT), Harbin 150001, China \\ ${ }^{2}$ Department of Computing, The Hong Kong Polytechnic University, Hung Hom, Kowloon, Hong Kong, China
}

Received 24 January 2005; Revised 9 September 2005; Accepted 12 September 2005

Recommended for Publication by William Sandham

Computerized pulse analysis based on traditional Chinese medicine (TCM) is relatively new in the field of automatic physiological signal analysis and diagnosis. Considerable researches have been done on the automatic classification of pulse patterns according to their features of position and shape, but because arrhythmic pulses are difficult to identify, until now none has been done to automatically identify pulses by their rhythms. This paper proposes a novel approach to the detection of arrhythmic pulses using the Lempel-Ziv complexity analysis. Four parameters, one lemma, and two rules, which are the results of heuristic approach, are presented. This approach is applied on 140 clinic pulses for detecting seven pulse patterns, not only achieving a recognition accuracy of $97.1 \%$ as assessed by experts in TCM, but also correctly extracting the periodical unit of the intermittent pulse.

Copyright ( 2006 Hindawi Publishing Corporation. All rights reserved.

\section{INTRODUCTION}

The quantification and analysis of physiological signals have become more important recently. The research on traditional Chinese pulse diagnosis (TCPD) is relatively new in this area. Usually, practitioners of TCPD use pulse sensors to acquire patients' pulse waveforms of the wrists, and then investigate the patients' pulse waveforms [1-7]. Presently, the long-term monitoring of pulse waveforms is becoming more popular. The automatic analysis and recognition of pulse waveforms are useful in reducing the heavy burden on practitioners of observing and analyzing pulse waveforms.

Many pattern recognition methods have been applied to the automatic recognition and classification of pulse waveforms. For example, Lee et al. applied fuzzy theory to analyze several cases of pulse waveforms and got good results [8]; Yoonet al. introduced three characteristics to describe a pulse: its position, its size, and its strength [9]; Stockmanet al. used structural pattern recognition to identify the shape of carotid pulse waveforms [10]; Wanget al. proposed an improved dynamic time warping algorithm for recognizing five pulse patterns that are distinct in their shapes [11]. Wang and Xiang applied a three-layer artificial neural network in order to recognize seven types of pulse patterns [12]. In all of these researches, only pulse patterns' features of position or shape are analyzed. We cannot find the research into differentiating pulse patterns according to their rhythms, yet the rhythm is a useful feature for identifying pulse patterns. The arrhythmic pulse patterns, which have distinctive rhythms, are difficult to recognize using their linear features. This paper presents an approach to the differentiation of the seven pulse patterns according to their rhythms. Four parameters were proposed to discriminate between rhythmic and arrhythmic pulses. We then applied the Lempel-Ziv complexity analysis in order to identify arrhythmic pulse patterns, achieving a total accuracy of $97.1 \%$.

This paper is organized as follows. Section 2 analyzes pulse rhythms. Section 3 proposes an approach based on Lempel-Ziv complexity analysis in order to recognize the characteristic rhythms of the seven pulse patterns. Section 4 discusses the experimental results. Section 5 offers conclusion.

\section{CLINICAL VALUE OF PULSE RHYTHM ANALYSIS}

TCPD recognizes that there are seven pulse patterns which have distinctive rhythms: four patterns are rhythmic and three patterns are arrhythmic. The four rhythmic pulse patterns are called swift pulse, rapid pulse, moderate pulse and Slow pulse. The three arrhythmic pulses are called running pulse, knotted pulse, and intermittent pulse. Figures $1(\mathrm{a})-1(\mathrm{~g})$ illustrate these pulses. In each figure, the first panel 
is the pulse waveform and its onsets and the second panel is its pulse interval series. Pulse interval (PI) is the interval between two consecutive onsets of pulse waveform.

Just as the heart rhythms identified using ECGs are important in Western medicine, these seven pulse patterns are important in TCPD [13]. They relate to syndromes identified in traditional Chinese medicine (TCM) and their specific behaviors closely guide diagnosis [14], see also http://www. itmonline.org/arts/pulse.htm. Swift pulse often occurs in severe acute febrile disease or consumptive conditions. Rapid pulse usually indicates the presence of heat. Moderate pulse reflects a normal condition of the body. Slow pulse often relates to endogenous cold. The running pulse feels rapid but loses a beat at irregular intervals, indicating blood stasis or the retention of phlegm. The knotted pulse feels leisurely but loses a beat at irregular intervals. The irregularity and slowness of this pulse are due to the obstruction of blood. The intermittent pulse, comparatively relaxed and weak, stops at regular intermittent intervals. It often occurs in exhaustion of viscera organs, severe trauma, or in moments of fright. The intermittent pulse periodically loses a beat after several but less than six normal PIs. Otherwise, the arrhythmic pulse may be either running or knotted pulse [13].

\section{THE APPROACH TO AUTOMATIC RECOGNITION OF PULSE RHYTHMS}

In Section 3.1, we will first outline the basic idea of LempelZiv complexity analysis. After that, we will introduce the definitions of four parameters, one lemma, and two rules in Section 3.2. Finally, we will describe our approach to recognizing the seven pulse patterns according to the different rhythms in Section 3.3.

\subsection{Lempel-Ziv complexity analysis}

Lempel-Ziv complexity analysis is an approach to evaluating the randomness of finite sequences. It is closely related to information entropy [15-18]. The Lempel-Ziv complexity measures the rate at which new patterns are generated in a symbolized sequence. It is based on a coarse-graining of the measurement, that is, the signal to be analyzed is transformed into a sequence made up of just a few symbols. Lempel-Ziv complexity measures the number of steps in a self-delimiting production process by which a given sequence is presumed to be generated. The complexity counter $c(n)$ measures the number of distinct patterns contained in a given sequence. Briefly, a sequence $S=s_{1} s_{2} s_{3} \cdots s_{n}$ (where $s_{1}, s_{2}$, etc. denote symbols, e.g., " 0 " or " 1 ") is scanned from left to right letter by letter and the $c(n)$ is increased by one unit when a new pattern of consecutive characters is encountered $[19,20]$.

The process of Lempel-Ziv complexity analysis is as follows. Let $Q$ and $R$ denote, respectively, subsequences of the sequence $S=s_{1} s_{2} s_{3} \cdots s_{n}$ and let $Q R$ be the concatenation of $Q$ and $R$, while subsequence $Q R D$ is derived from $Q R$ after its last character is deleted ( $D$ means the operator to delete the last character in a sequence). Let $L(Q R D)$ denote the lexicon of all different patterns of $Q R D$. In the beginning, $c(n)=1, Q=s_{1}, R=s_{2}$, therefore, $Q R D=s_{1}$. Now assume that $Q=s_{1} s_{2} s_{3} \cdots s_{i}$, and $R=s_{i+1}$, then $Q R D=s_{1} s_{2} s_{3} \cdots s_{i}$. If $R \in L(Q R D)$, that is, $R$ is a subsequence of $Q R D$, then $R$ is not a new pattern. At this time, $Q$ need not change and renew $R$ to be $s_{i+1} s_{i+2}$. After that, we judge whether $R$ belongs to $L(Q R D)$ and continue until $R \notin L(Q R D)$. If $R=s_{i+1} s_{i+2} \cdots s_{i+j}$ is not a subsequence of $Q R D=s_{1} s_{2} s_{3} \cdots s_{i+j-1}$, increase $c(n)$ by one. Thereafter, combine $Q$ with $R$ and renew $Q$ to be $s_{1} s_{2} s_{3} \cdots s_{i+j}$. At the same time, renew $R$ to be $s_{i+j+1}$. Repeat these processes until $R$ is the last character in the sequence $S$. Thus, the number of different patterns is $c(n)$, that is, the measure of complexity. Ziv and Lempel insert slashes into the sequence $S$ at the position where a new pattern occurs. Thus, they divided the sequence $S$ into $c(n)$ blocks using those slashes.

\subsection{Definitions and basic facts}

To recognize pulse patterns with different rhythms, we first extract four parameters defined in Definitions 1 and 2. The parameters in Definition 1 are extracted from PI series and are used to judge if the pulse waveform is arrhythmic. If the pulse waveform is arrhythmic, we need to symbolize its PI series. The parameters in Definition 2 are extracted from symbolized pulse intervals (SPIs), which are obtained by the coarse-graining technique, and then they are used to judge if the pulse waveform is an intermittent pulse.

\subsubsection{Definitions}

Definition 1. Assume that $T=$ " $t_{1}, t_{2}, \ldots, t_{N}$ " is a PI series. To judge whether its corresponding pulse is arrhythmic or not, define two parameters.

\section{Variation range (VR)}

$\mathrm{VR}$ is the difference between the maximum element and minimum element of $T$, that is,

$$
V R=\max (T)-\min (T)
$$

\section{Variation coefficient (VC)}

$\mathrm{VC}$ is the ratio between standard deviation and the average of this series $T$,

$$
\mathrm{VC}=\frac{\mathrm{SD}}{\bar{t}} \times 100 \%
$$

where

$$
\bar{t}=\frac{1}{N} \sum_{i=1}^{N} t_{i}, \quad S D=\sqrt{\frac{\sum_{i=1}^{N}\left(t_{i}-\bar{t}\right)^{2}}{N-1}} .
$$

Definition 2. Assume that $S=$ " $s_{1} s_{2} \cdots s_{N}$ " is a SPI sequence. To determine whether an arrhythmic pulse is an intermittent pulse or not, define two parameters as follows. 

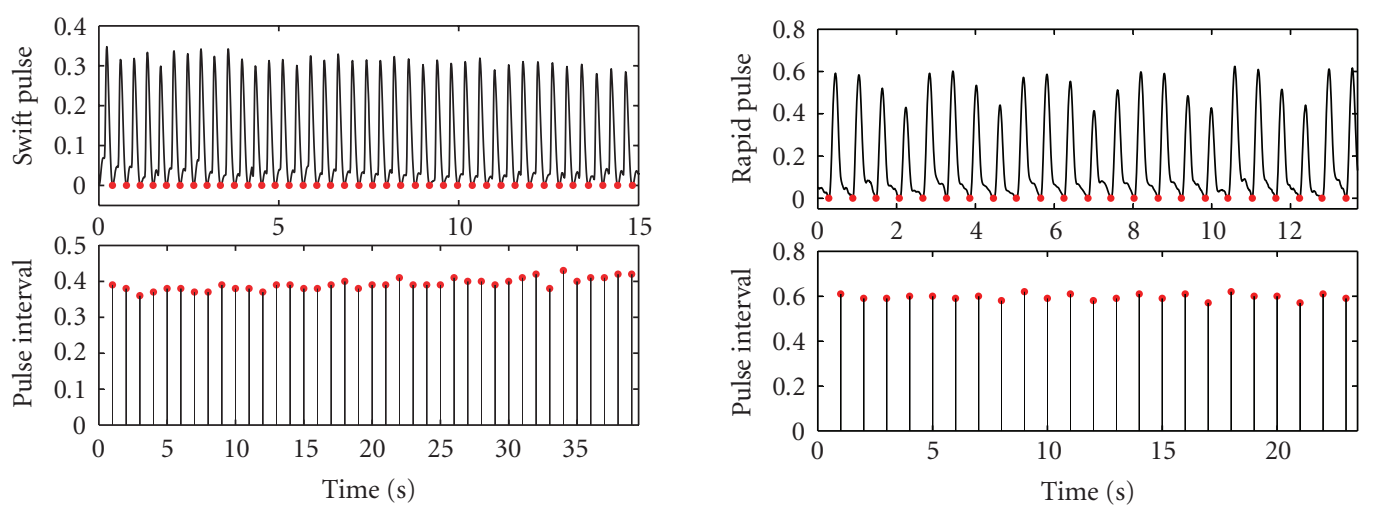

(a)

(b)
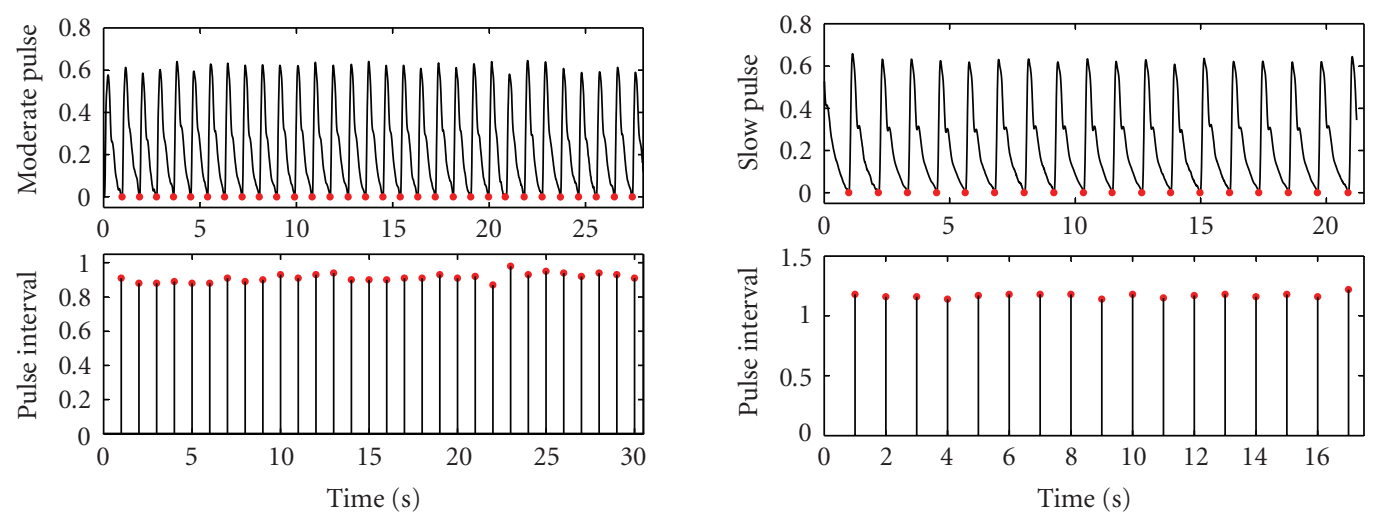

(c)

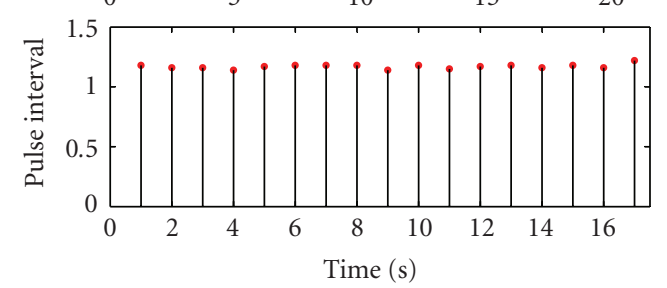

(d)
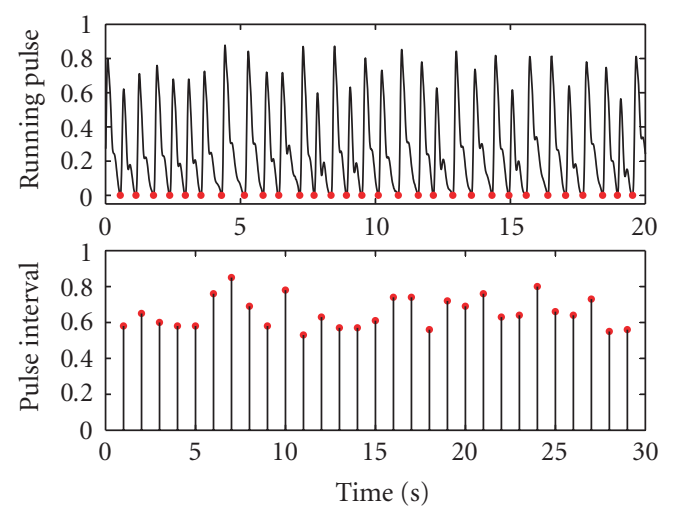

(e)
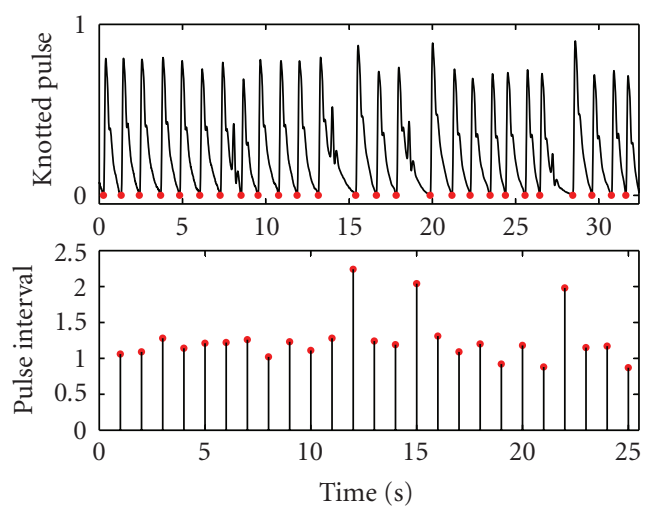

(f)

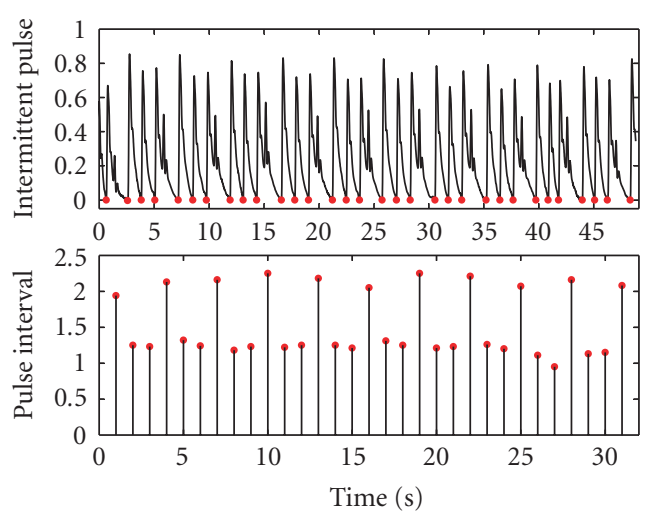

(g)

FIGURE 1: The seven pulse waveforms, which are distinct in rhythms: (a) swift pulse; (b) rapid pulse; (c) moderate pulse; (d) slow pulse; (e) running pulse; (f) knotted pulse; (g) intermittent pulse. 


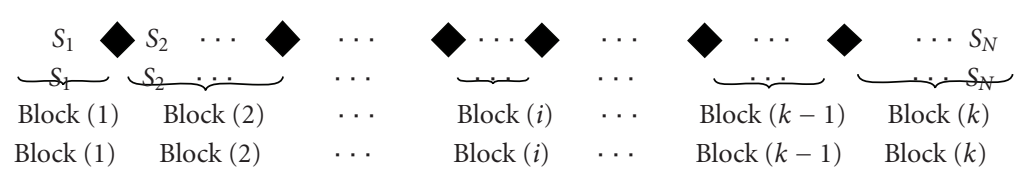

Figure 2: Result of the Lempel-Ziv complexity analysis of one sequence. We insert " $\diamond$, where a new pattern emerges according to the Lempel-Ziv complexity analysis. Here, the $k$ "s divide the sequence " $S_{1} S_{2} \cdots S_{N}$ " into $k$ blocks.

\section{Minimum recurrent unit (MRU)}

MRU is the subsequence that is the minimum periodic unit of the sequence $S$.

\section{Recurrent degree (RD)}

$\mathrm{RD}$ is the recurrent time of a finite sequence $S$. The RD = $\lfloor\mathrm{L} / \mathrm{L} r\rfloor$, where $L r$ is the length of its MRU; $L$ is the length of the sequence $S$. That is to say RD is the largest integer, which does not exceed the value of $L$ divided by $L r$.

For examples, the sequence " 1234005 " is a nonperiodic sequence, whose MRU is itself " 1234005 " and whose RD = $\lfloor 7 / 7\rfloor=1$; the sequence " 1212121 " is a periodic sequence, whose MRU is " 12 " and whose $\mathrm{RD}=\lfloor 7 / 2\rfloor=3$.

\subsubsection{Rules}

To differentiate pulse rhythms, we offer two rules which combine the experience of experts in TCPD with the Lempel-Ziv complexity analysis. According to Rule 1 , the rhythmic pulses and arrhythmic pulses can be differentiated. According to Rule 2, the intermittent pulse can be differentiated from the running pulse and the knotted pulse.

Given the VC and VR of a PI series, it is possible to determine whether the pulse is arrhythmic according to Rule 1. If the pulse is arrhythmic, we need to symbolize the PI series using coarse-graining method. We then extract the subsequences from SPI series and simplify those subsequences further (the simplification process will be discussed in Section 3.3.4).

The intermittent pulse periodically has one pause after several normal beats. The number of consecutive normal beats must be less than 6 and constant. Thus, we scan the SPI sequence from leftmost to rightmost and extract several subsequences that begin with first symbol "1" and end at symbol "1" which has at least six continuous "0"s on its right or is the rightmost symbol " 1 " of this whole sequence. For example, “00001001000000100000100101000100000000001000" is a symbolized pulse interval series. The extraction of its subsequences can be "0000\#1001\$000000\#10000010010 $10001 \$ 0000000000 \# 1 \$ 000$." The symbols “\#” and "\$” stand for the beginning and the end of the subsequence we extracted, respectively. Here, Subsequence1 ="1001," Subsequence2 =“1000001001010001," Subsequence3 =“1."

Rule 1. If the VC of a PI series is greater than $20 \%$ or the VR of a PI series is more than the second minimum of this PI series, the pulse corresponding to this PI series is an arrhythmic pulse.
Rule 2. After the coarse graining, subsequences extraction and simplification processes, we can obtain the symbolized subsequences of the original PI series. If the RD of a symbolized subsequence is equal to or more than three, its corresponding pulse is an intermittent pulse [13].

Rule 2 requires that the symbolized subsequences of an intermittent pulse be periodic and contain at least three periods because just having two periods could be a random phenomenon and should not be taken as regularity. Consequently, the problem of differentiating the intermittent pulse from the knotted pulse and the running pulse is equivalent to judging whether a subsequence $S_{\text {sub }}$ is a periodic subsequence with at least three periods. This kind of periodic symbolized subsequences has special characteristics described in the following lemma.

\subsubsection{Lemma}

Lemma 1. Assume that a periodic symbolized subsequence $S_{\text {sub }}=s_{1} s_{2} \cdots s_{N}$ contains at least three periods and the length of its MRU is $P$. Ziv and Lempel insert delimiters into the subsequence to be analyzed using the two rules they defined $[15,16]$. These delimiters divide a subsequence into several blocks. In Figure 2, insert " " to divide the subsequence into $k$ blocks. It will be proved that the Lempel-Ziv complexity analysis result of periodic subsequence $S_{\text {sub }}$, which contains at least three periods, must satisfy the following five inequalities:

(1)

$$
P \leq \frac{1}{3} \sum_{i=1}^{k}|\operatorname{Block}(i)|
$$

(2)

$$
P>\sum_{i=1}^{k-2} \mid \text { Block }(i) \mid \text {; }
$$

(3)

$$
P \geq|\operatorname{Block}(i)|, \quad i=1, \ldots, k-1 ;
$$

$$
2 P>\sum_{i=1}^{k-1}|\operatorname{Block}(i)| \geq P ;
$$

$$
\mid \text { Block }(k) \mid>P,
$$




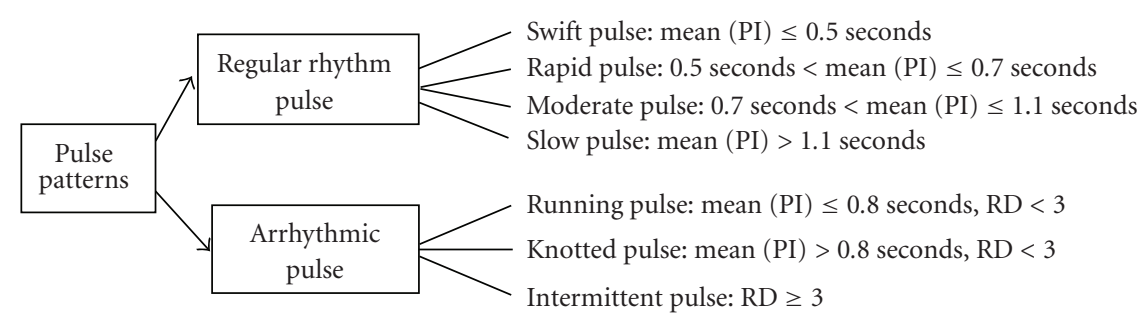

FIGURE 3: The characteristics of seven pulse patterns, which differ in rhythm. PI is the abbreviation of pulse interval. The mean (PI) stands for the average of PI series. RD is the abbreviation of recurrent degree.

where $\mid$ Block $(i) \mid$ is the length of the ith block. In the following, these five inequalities (4)-(8) will be proved.

Proof. (1) According to the premise, the subsequence $S_{\text {sub }}$ is periodic and contains at least three periods. Thus, $\sum_{i=1}^{k}|\operatorname{Block}(i)| \geq 3 P$, that is, $P \leq(1 / 3) \sum_{i=1}^{k}|\operatorname{Block}(i)|$.

(2) If $\sum_{i=1}^{k-2} \mid$ Block $(i) \mid \geq P$, the former $k-2$ blocks must contain at least one MRU. Then, Block $(k-1)$ and Block $(k)$ must repeat the former patterns because subsequence $S_{\text {sub }}$ is a periodic subsequence which contains three periods at least. Thus, Block $(k-1)$ and Block $(k)$ cannot be segmented into two blocks according to Lempel-Ziv complexity analysis. Therefore, $P>\sum_{i=1}^{k-2}|\operatorname{Block}(i)|$.

(3) According to (5), we know that $P \geq|\operatorname{Block}(i)|, i=$ $1, \ldots, k-2$. Thus, we only need to proved $P \geq \mid$ Block $(k-1) \mid$. Assume that $P<\mid$ Block $(k-1) \mid$, then Block $(k-1)$ contains more than one MRU. In (5), $P>\sum_{i=1}^{k-2}|\operatorname{Block}(i)|$, the first $P$ symbols of Block $(k-1)$ must be a new pattern, which is different from the first $k-2$ blocks. Therefore, Block $(k-1)$ must be divided into several blocks according to Lempel-Ziv complexity analysis. However, Block $(k-1)$ is the $(k-1)$ th Block. Thus, $P \geq|\operatorname{Block}(i)|, i=1, \ldots, k-1$.

(4) If $P>\sum_{i=1}^{k-1} \mid$ Block $(i) \mid$, the first $P-1$ symbols of Block $(k)$ must be a new pattern, otherwise the length of the MRU of $S$ is less than $P$, contradicting the assumption. Thus, $\sum_{i=1}^{k-1}|\operatorname{Block}(i)| \geq P$. According to (5), if $\sum_{i=1}^{k-1}|\operatorname{Block}(i)| \geq$ $2 P$, the length of Block $(k-1)$ must be larger than $P$. However, the first $P-1$ symbols of Block $(k-1)$ must be a new pattern, that is, the length of Block $(k-1)$ should be less than $P-1$, contradicting (6). Therefore, we draw the conclusion that $2 P>\sum_{i=1}^{k-1}|\operatorname{Block}(i)| \geq P$.

(5) According to (4) and (5), that is, $\sum_{i}=1^{k-1}|\operatorname{Block}(i)|<$ $2 P$ and $\sum_{i=1}^{k}|\operatorname{Block}(i)| \geq 3 P$, we can prove that $\mid$ Block $(k) \mid$ $>P$.

\subsubsection{The seven pulse patterns' characteristics in rhythms}

Figure 3 illustrates the rhythmic characteristics of these seven pulse patterns. The swift, rapid, moderate and slow pulses are rhythmic pulses and are differentiated by the average of their PIs. The knotted, running, and intermittent pulses are arrhythmic pulses and their SPIs have different RDs. The intermittent pulse has periodic arrhythmia, and the RD of the symbolized intermittent pulse interval sequence is higher than 2. The RDs of both the knotted pulse and the running pulse are less than 3. Additionally, the PI average of the knotted pulse is longer than that of the running pulse.

\subsection{Automatic recognition of pulse patterns distinctive in rhythm}

Essentially, TCM practitioners identify pulse rhythms in three steps. First, they identify the average of PI series. Second, they identify the variation of PI series and judge if the pulse is arrhythmic or not. Finally, if the pulse is arrhythmic, they must ascertain whether the irregular rhythm is periodic.

Figure 4 outlines our approach to the automatic recognition of these seven pulse patterns. The pulse waveform, which is easy to be distorted by noise and baseline wander, must be preprocessed firstly. We then extract the PI series and calculate the VC, VR, and the average of this PI series and judge if this PI series is arrhythmic. The PI series will be symbolized and the subsequences that contain the abnormal PI will be extracted. After that, we simplify the extracted subsequences. Next, the Lempel-Ziv complexity analysis method is used to analyze the extracted symbolized subsequences. Finally, we judge if the symbolized subsequences are periodic according to the lemma and Rule 2. Thus, the seven pulse patterns can be automatically differentiated.

\subsubsection{Preprocessing the pulse waveform}

The pulse waveform should be preprocessed before being analyzed because noise, respiration, and artifact motion can be introduced during pulse waveform acquisition. It is important to remove the pulse waveform's baseline drift and attenuate noise before the automatic analysis of pulse waveforms. First, we filtered the power-line interference at $50 \mathrm{~Hz}$ and then applied wavelet approximation to estimate the baseline wander of pulse waveform [21]. After that, the signal-tonoise ratio of the pulse waveform is greatly enhanced; thus, the accurate extraction of PI series in the following step is assured.

\subsubsection{Pulse interval extraction and calculation of its VC and VR}

In order to analyze the rhythm of the pulse waveform, we first extract the PI series of pulse waveform and then calculate its VC and VR. Many algorithms have been previously 


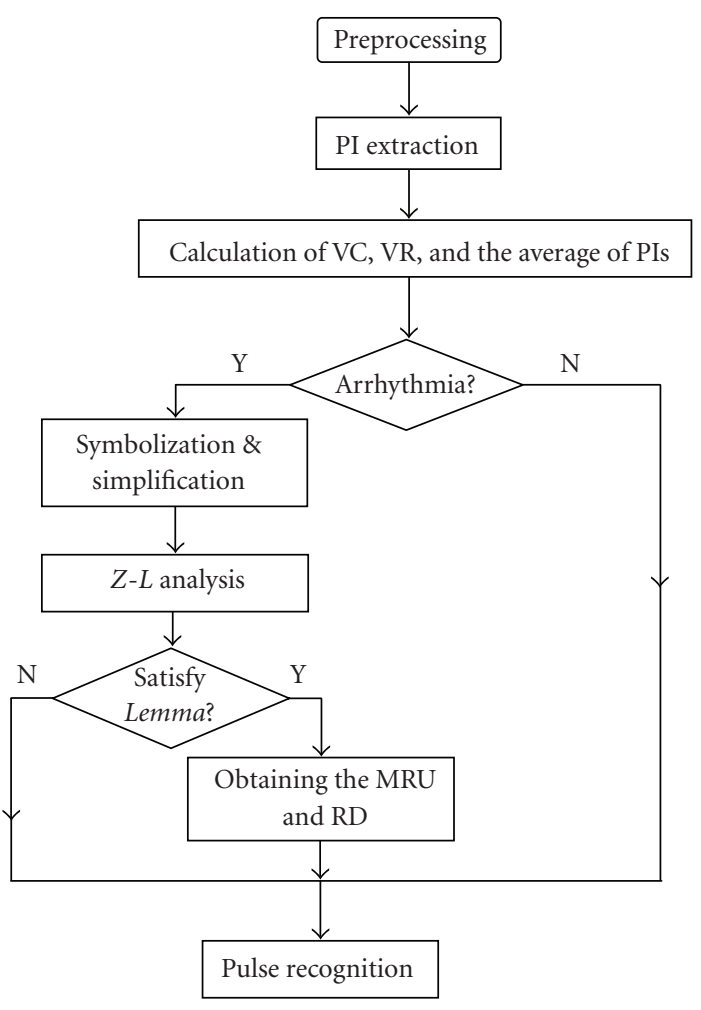

FIgURE 4: Our approach to the differentiation of the seven rhythmically distinct pulse patterns.

proposed for the accurate detection of the intervals between the beats of a blood pressure waveform [22-24]. Here, we use the method in [25] to detect the onsets of pulse waveform.

In order to further explain the calculation of the VC, VR, and Rule 1, we take Figures 5 and 6 as examples. Figure 5(a) shows a slow pulse, whose VR $=(1.41-1.12)<1.20$ seconds and $\mathrm{VC}=8 \%<20 \%$ (where the maximum, minimum, the second minimum, and the average of PI series are 1.41, 1.12, 1.20 , and 1.26 seconds, resp.). In Figure 5(b), the pulse is arrhythmic and its VR $=(1.92-0.90)>0.96$ seconds (where the maximum, minimum and the second minimum of PI series are $1.92,0.90$, and 0.96 seconds, resp.). Figure 7 shows, the 157 PIs of the 200-second pulse waveform in Figure 6. Its $\mathrm{VR}=2.25-0.86=1.39>0.91$ and its VC is $25 \%$, illustrating that this 200 second pulse is arrhythmic according to Rule 1 (where the maximum, the minimum, and the second minimum of PI series are 2.25, 0.86, and 0.91 seconds, resp.).

\subsubsection{Symbolizing pulse interval series and subsequence extraction}

To classify the pulse pattern of an arrhythmic pulse, we analyze the distribution of the PI series and then symbolize this PI series according to its distribution. It can be imagined that the histogram of an arrhythmic PI series must contain two peaks with a valley between them: the first peak corresponds to the normal interval and the second corresponds to the abnormal interval. We define $T_{a}$ as the PI corresponding to the first peak in the leftmost of the PI histogram, $T_{b}$ as the PI corresponding to the second peak of the PI histogram. We then define $T_{\text {sym }}$ as $\left(T_{b}+T_{a}\right) / 2$. If the PI is higher than Tsym, it is symbolized as "1," otherwise it is symbolized as "0." Figure 8 shows the histogram of the PIs extracted from the pulse waveform in Figure 6. Here, $T_{a}=1.14$ seconds, $T_{b}=2.19$ seconds, $T_{\text {sym }}=\left(T_{b}+T_{a}\right) / 2=1.67$ seconds, as demonstrated in Figures 7 and 8. Thus, the SPI of Figure 6 is as follows.

$$
\begin{aligned}
& \text { SPI } \\
& \text { = "000010010010010010010010000000000000 } \\
& 0000000000000000100000000000000000100 \\
& 1001001001001001001001001001001000000 \\
& 000000000000000000000000000000000000 \\
& \text { 0000000000(length = 157)." }
\end{aligned}
$$

Usually, the PIs are normal. Abnormal PIs occur only occasionally but should receive considerable attention in automatic pulse rhythm analysis because they are related to the disorder of cardiovascular system. we search the SPI sequence from left to right and then extract the subsequences that start from the first symbol " 1 " and end at the symbol " 1 " which is followed by at least six continuous " 0 "s or is the rightmost " 1 " of the sequence. This process is repeated until the whole sequence has been searched.

Equation (10) illustrates the extraction result of the SPI in Figure 6. The symbols "\#” and "\$" stand for the start and the end of the subsequence we extracted, respectively,

$$
\begin{aligned}
& \text { SPI } \\
& =0000 \# 1001001001001001001 \$ 000000000000 \\
& \quad 00000000000000000 \# 1 \$ 00000000000000000 \\
& \quad \# 1001001001001001001001001001001001 \$ 0 \\
& \quad 0000000000000000000000000000000000000 \\
& \\
& 000000000000000(\text { length }=157) .
\end{aligned}
$$

From (10), we extracted three subsequences illustrated in (11), (12), and (13):

$$
\begin{gathered}
\text { Subsequence1 = "1001001001001001001," } \\
\text { Subsequence } 2=\text { " } 1, "
\end{gathered}
$$

Subsequence3 =“1001001001001001001001001001001001."

\subsubsection{Arrhythmic pulse recognition based on Lempel-Ziv complexity analysis}

Intermittent pulse is a special kind of arrhythmic pulse because its arrhythmia is periodical. Thus, after symbolizing the PI series and extracting subsequences of the SPI sequence, the recognition of the intermittent pulse is equivalent to judging if the symbolized subsequences are periodic subsequences that contain at least three periods. Hence, we can differentiate intermittent pulse using the 


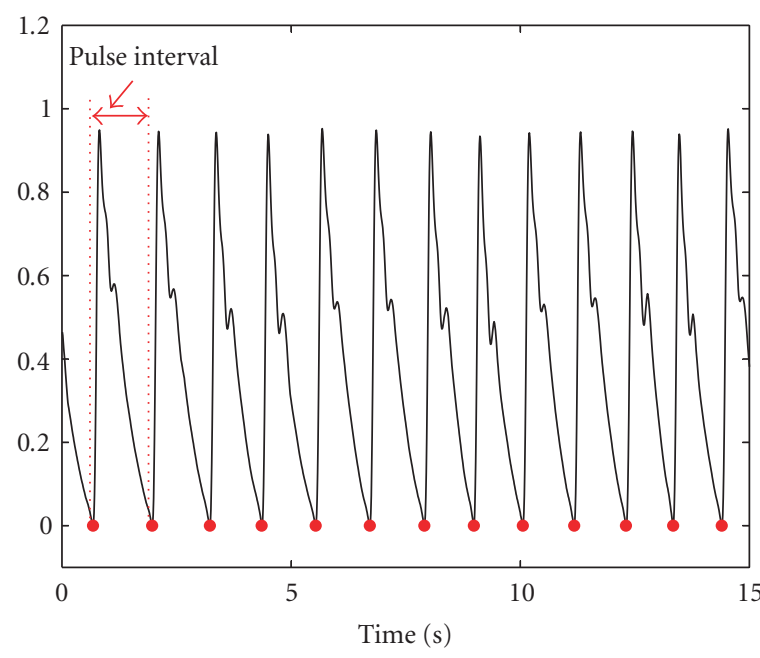

- Pulse wave

- Pulse onset

(a)

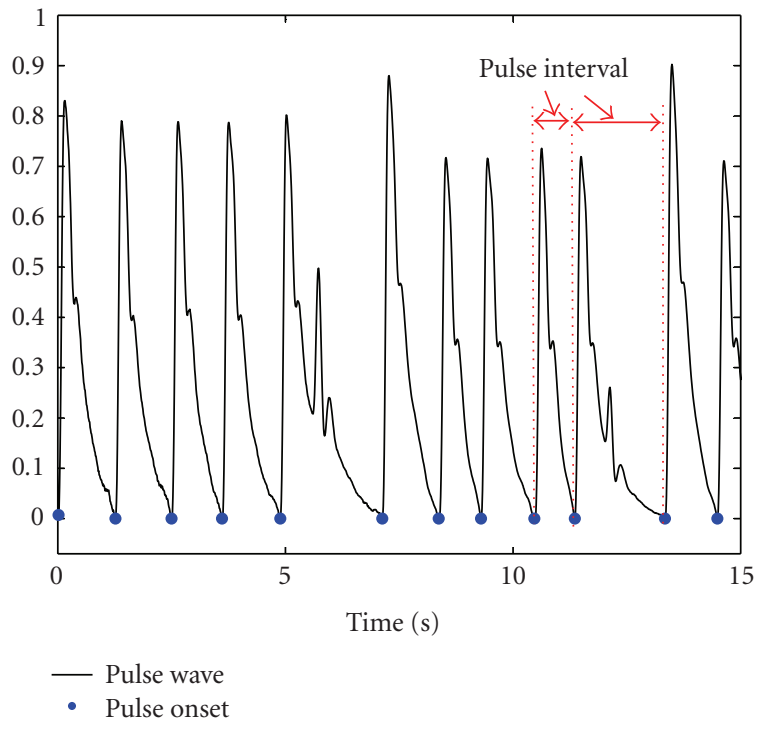

(b)

FIGURe 5: Pulse onsets and PI series: (a) the pulse waveform with normal rhythm; (b) the pulse waveform with abnormal rhythm.

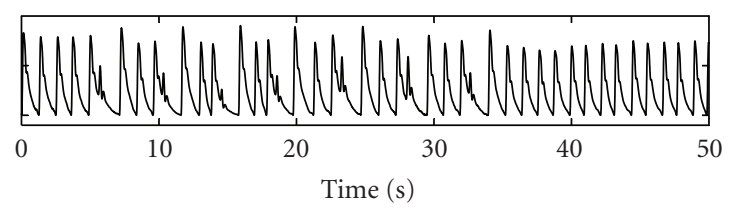

(a)

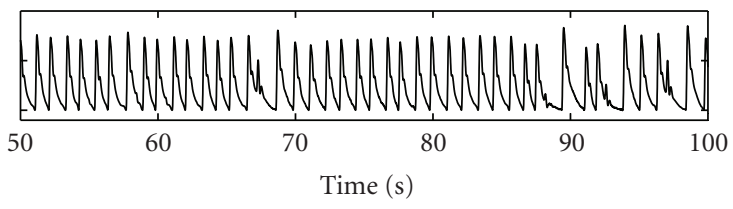

(b)

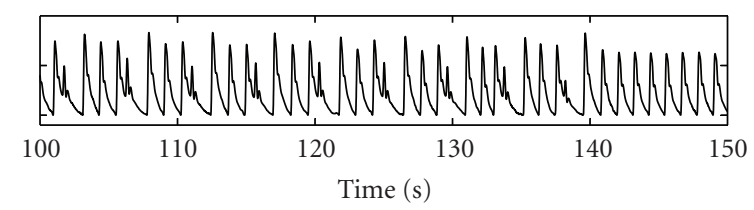

(c)

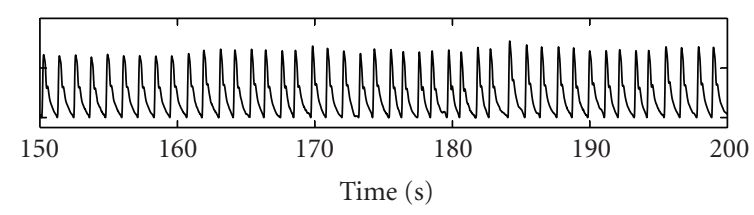

(d)

FIgURE 6: Arrhythmic pulse of 200 seconds (157 pulse periods).

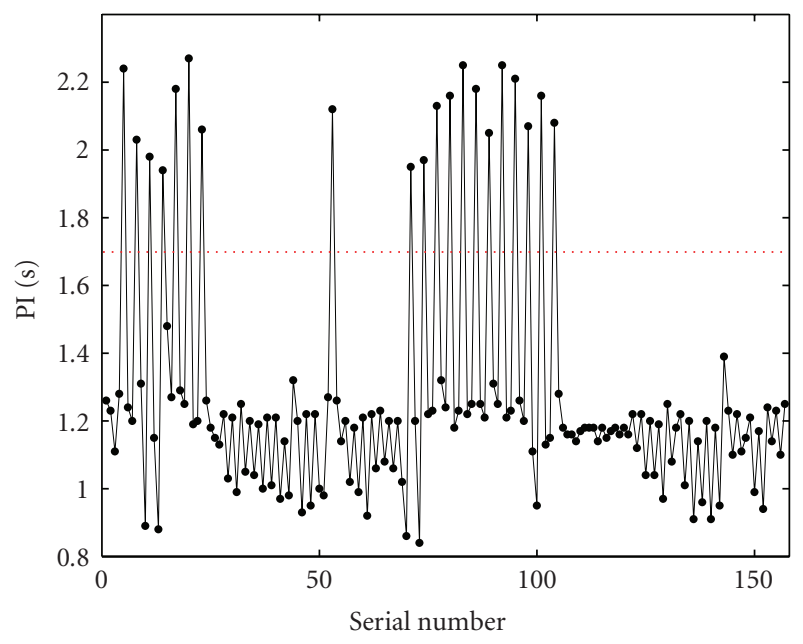

FIGURE 7: Pulse intervals of a 200-second arrhythmic pulse; $T_{\text {sym }}=$ 1.67 .

string-matching method. The MRU of an intermittent pulse might be in the form of (a) basic form: $10^{i}\left(0^{i}\right.$ represents $i$ consecutive 0 's), $0 \leq i \leq 5$; or (b) composite form: combinations of the basic forms, such as $10^{i} 10^{j}(0 \leq i \leq 5$, $0 \leq j \leq 5$, and $j \neq i$ ), and so on. For example, " 10 " is the MRU of sequence " 101010101 "; "100" is the MRU of sequence "1001001001001"; "10100" is the MRU of sequence “101001010010100101001.” However, Lempel-Ziv analysis can split the basic form $10^{i}$. Thus, it will cause damage to the actual purpose of searching MRU. 


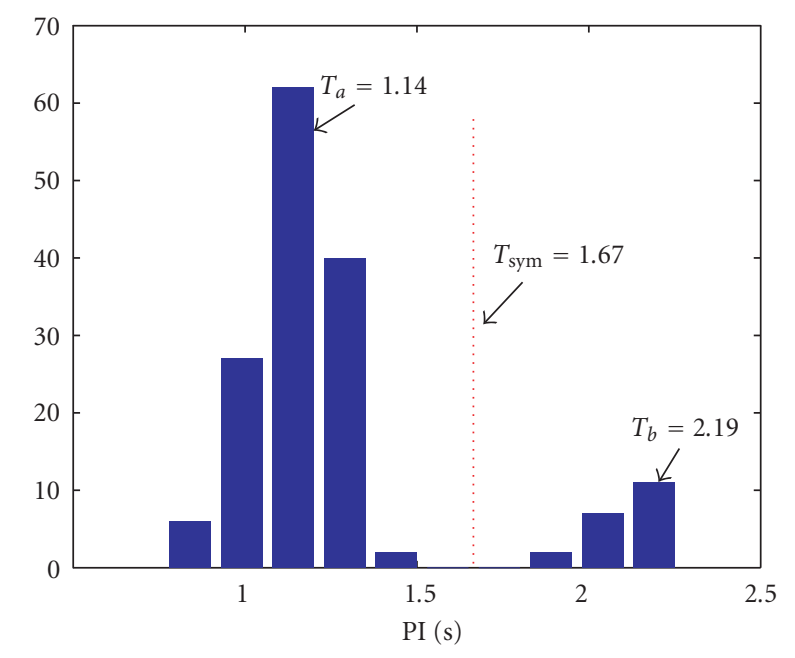

FIgURE 8: Histogram of PIs. $T_{a}, T_{b}, T_{\text {sym }}$ are also demonstrated here.

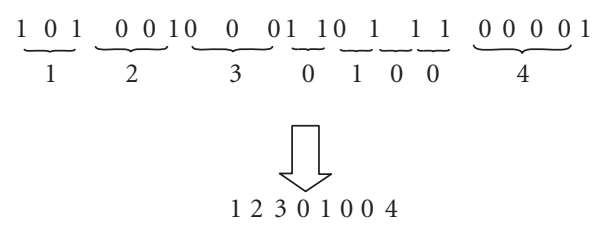

Figure 9: An example of simplification. The new simplified sequence denotes the number of "0"s between two nearest " 1 "s. For sequence " $10^{i} 1$ " $(0 \leq i \leq 5)$, we denote it as " $i$." If two " 1 "s are consecutive, there is no " 0 " between these two " 1 "s. Thus, we denote "0." In this figure, we scan the SPI " 10100100011011100001 " from left to right. Between the first " 1 " and the second " 1 ," there is one zero; between the second " 1 " and the third " 1 ," there are two zeros. Repeat this procedure until the last " 1 ." We can simplify the SPI into "12301004."

\section{A. Simplification of symbolized pulse interval sequence}

To prevent from splitting the basic form $10^{i}$, we further simplify the binary SPI subsequences. We denote the basic form of the recurrent unit numerically by letting $i$ denote $10^{i} 1$, $0 \leq i \leq 5$. That is to say, $i$ denotes the number of successive " 0 "s between the two nearest " 1 " $s$. If two nearest " 1 "s are conjoint, the number of successive " 0 "s between these two nearest " 1 "s is 0 . Thus, the original sequence can be simplified into a new sequence constituted by these " $i$ "s. For example, the sequence " 10100100011011100001 " can be expressed as "12301004" illustrated in Figure 9.

Thus the Subsequence1 and Subsequence3 in (11) and (13) can be simplified as

$$
\begin{gathered}
\text { Subsequence1 = "222222,” } \\
\text { Subsequence3 = "22222222222.” }
\end{gathered}
$$

The Subsequence2 in (12) is only one symbol "1," whose RD $=1$ is obvious.

\section{B. Lempel-Ziv complexity analysis of simplified pulse interval sequence}

Assume a sequence $S=s_{1} s_{2} \cdots s_{N}$. To indicate a substring of $S$ that starts at position $i$ and ends at position $j$, we denote it as $S(i, j), i \leq j$. Q is called a prefix of $S$ if there exists an integer $i$ such that $Q=S(1, i), 1 \leq i<N$.

One simple method for determining whether a symbolized subsequence is a periodic sequence that contains at least three periods is to assume that each of the prefixes of $S$ is the MRU and then to match it with the remaining part of $S$. We call this method naïve matching. If $S$ is a periodic sequence with at least three periods, this method requires $O(n)$ time, where $n$ represents the length of the sequence $S$. If $S$ is not a periodic sequence with at least three periods, this method requires $O\left(n^{2}\right)$ time to make the conclusion, which is time consuming.

Considering the time consuming of naïve matching, we proposed a matching method based on Lempel-Ziv complexity, which generally requires $O(n)$ time to make the conclusion whether $S$ is a periodic sequence with at least three periods or not. Having simplified the expression of the SPI sequence, we analyze Subsequence1 and Subsequence3 in (14) using Lempel-Ziv complexity analysis. During the analysis, when a new pattern emerges, the symbol " $\$$ " is inserted after it. The complexity analysis result of Subsequencel is as follows.

(1) The first character is always a new pattern. Therefore, the first pattern is $\rightarrow 2$

(2) The second character is " 2 " and this is identical to the first pattern. In this case, the old pattern also contains " 2 ," so it is not a new pattern. The analysis result is $\rightarrow 2 \checkmark 2$.

(3) The third character is "2." The current pattern is " 22. " The previous patterns are " 2 " and " 22 ," so " 22 " still is not a new pattern and can be marked as $\rightarrow 2 \downarrow 22$.

(4) Repeating this process, this sequence is segmented into two blocks:

$$
\text { Subsequence1 = "2 } 22222 \text {." }
$$

The complexity analysis of Subsequence3 is similar to the analysis of Subsequence1. Its Lempel-Ziv complexity analysis result is “ 2222222222 ."

\section{Judging whether the arrhythmic pulse is an intermittent pulse}

Having analyzed the Lempel-Ziv complexity of the SPI series, we must judge whether the subsequence is a periodic subsequence which contains at least three periods. Our approach consists of two phases.

Phase 1. Exclude the subsequences that could not satisfy the lemma.

The Lempel-Ziv complexity analysis separates $S_{\text {sub }}$ into $k$ blocks. If the Block $(k)$ is a new pattern, this subsequence must be nonperiodic. Furthermore, the length of each block ( $\operatorname{Block}(i) \mid, 1 \leq i \leq k)$ is obtained. If the Block $(k)$ is not a new pattern, replace the variables in (4), (5), (6), (7), and 


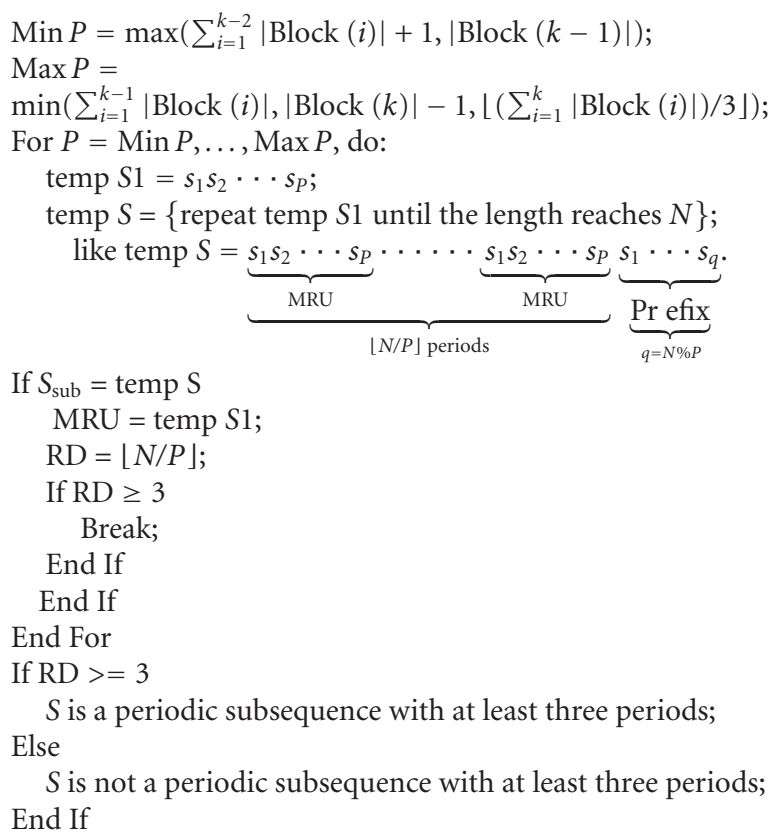

$S$ is a periodic subsequence with at least three periods; Else

$S$ is not a periodic subsequence with at least three periods; End If

\section{Algorithm 1}

(8) of the lemma with the actual values to see whether the inequalities can be met simultaneously. If the answer is yes, continue the steps described in the second phase; otherwise, $S_{\text {sub }}$ is not a periodic subsequence with at least three periods.

Phase 2. Further determine whether the subsequences that satisfy the lemma are the periodic subsequences with at least three periods.

In Phase 2, we first estimate the range of the MRU's length $P$ according to (4)-(8). According to Rule 2, we then further judge if this subsequence is a periodic subsequence with at least three periods. If the answer is yes, we will extract the MRU of this subsequence and compute its RD. Assume that $S_{\text {sub }}=s_{1} s_{2} \cdots s_{N}$, the algorithm of the second phase is shown in Algorithm 1.

In Algorithm 1, we first compute the range of the MRU's length $P$ according to (4)-(8). In the "For" loop, several periodic sequences are generated, with each one corresponding to a possible value of $P$, and these periodic sequences are matched with $S_{\text {sub }}$. In this process, the MRU and RD can be obtained at the same time. If $\mathrm{RD}<3, S_{\text {sub }}$ is not a periodic subsequence with at least three periods and its corresponding pulse is not an intermittent pulse.

From the Lempel-Ziv analysis results of Subsequence1 and Subsequence3, we find that the Subsequence1 and Subsequence 3 satisfy the inequalities of the lemma. Thus, we use the algorithm in Phase 2 to obtain the MRU and RD. The MRU of both Subsequence1 and Subsequence3 is " 100 ." The length of Subsequence1 and Subsequence3 are 19 and 34, respectively. The RDs of Subsequence1 and Subsequence3 are $\lfloor 19 / 3\rfloor=6$ and $\lfloor 34 / 3\rfloor=11$ respectively. Thus, we can offer
TABle 1: Comparison of matching times of Lempel-Ziv-analysisbased matching method and naïve matching method.

\begin{tabular}{|c|c|c|c|c|c|}
\hline \multirow{2}{*}{\multicolumn{2}{|c|}{ Symbolized sequence }} & \multirow{2}{*}{$\operatorname{Min} P$} & \multirow{2}{*}{$\operatorname{Max} P$} & \multicolumn{2}{|c|}{ Times of matching } \\
\hline & & & & Lempel-Ziv & Naïve \\
\hline \multirow{5}{*}{$\begin{array}{l}\mathrm{RD} \geq \\
3\end{array}$} & $(1)^{10}$ & 1 & 1 & 1 & 1 \\
\hline & $(12)^{10}$ & 2 & 2 & 1 & 2 \\
\hline & $(123)^{10}$ & 3 & 3 & 1 & 3 \\
\hline & $(1234)^{10}$ & 4 & 4 & 1 & 4 \\
\hline & $(4131123)^{10}$ & 7 & 7 & 1 & 7 \\
\hline \multirow{4}{*}{$\begin{array}{l}\mathrm{RD}= \\
2\end{array}$} & 11121112 & 3 & 2 & 0 & $\lfloor 8 / 3\rfloor=2$ \\
\hline & 111211121 & 3 & 3 & 1 & $\lfloor 9 / 3\rfloor=3$ \\
\hline & 1112111211 & 3 & 3 & 1 & $\lfloor 10 / 3\rfloor=3$ \\
\hline & 11121112111 & 3 & 3 & 1 & $\lfloor 11 / 3\rfloor=3$ \\
\hline \multirow{5}{*}{$\begin{array}{l}\mathrm{RD}= \\
1\end{array}$} & $1234(1)^{6}$ & 5 & 3 & 0 & $\lfloor 10 / 3\rfloor=3$ \\
\hline & $1234(12)^{22}$ & 5 & 7 & 3 & $\lfloor 48 / 3\rfloor=16$ \\
\hline & $(123)^{7} 1234$ & 21 & 0 & 0 & $\lfloor 25 / 3\rfloor=8$ \\
\hline & $12(1)^{10} 13$ & 10 & 0 & 0 & $\lfloor 14 / 3\rfloor=4$ \\
\hline & $(123)^{10} 13$ & 28 & 0 & 0 & $\lfloor 32 / 3\rfloor=10$ \\
\hline
\end{tabular}

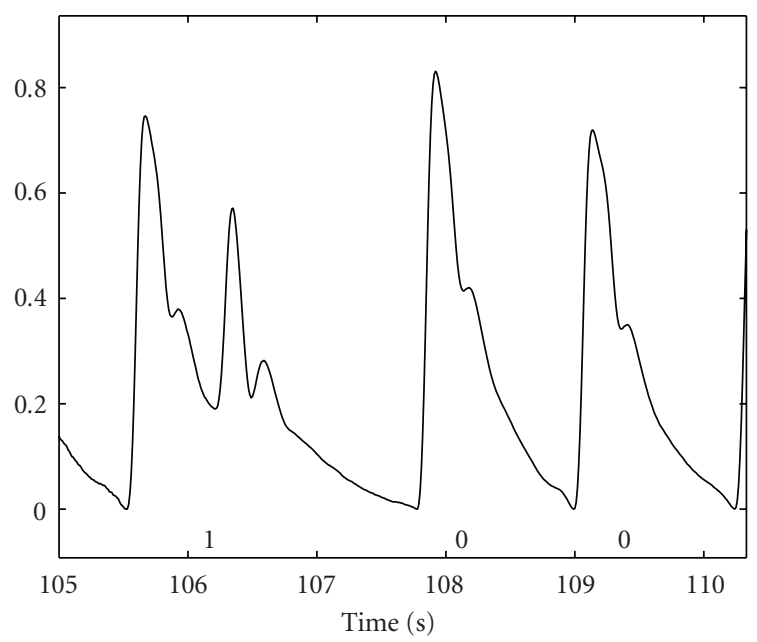

FIgURE 10: The MRU of the arrhythmic pulses in Figure 6.

a conclusion that this pulse is an Intermittent pulse, whose MRU is demonstrated in Figure 10.

Our Lempel-Ziv-complexity-based matching method is faster than the naïve matching method. The Lempel-Ziv complexity analysis is $O(n)$ time algorithm [26]. After the Lempel-Ziv complexity analysis, we exclude many subsequences that could not satisfy the inequalities in the lemma. Thus, our approach takes nearly the same time as LempelZiv complexity analysis. If $S_{\text {sub }}$ cannot be excluded, this subsequence can be further analyzed in Phase 2. Our approach usually needs to match only two or three times after estimating the range of the MRU's length. Thus, no matter whether $S_{\text {sub }}$ is a periodic subsequence or not, our approach takes $O(n)$ time to judge whether $S_{\text {sub }}$ is a periodic subsequence with at least three periods or not. Table 1 compares the matching times using Lempel-Ziv analysis method and the 
TABLE 2: Results of Lempel-Ziv-complexity-based matching approach.

\begin{tabular}{|c|c|c|c|c|}
\hline Pulse & Lempel-Ziv analysis result & Satisfy the lemma? & MRU & $\mathrm{RD}$ \\
\hline \multirow[t]{3}{*}{ Pulse1 } & "2" & No & “1000001001010001” & 1 \\
\hline & “ $5 \gg 2 \diamond 1 \diamond 3$ & No & “1001” & 1 \\
\hline & “1” & No & “1” & 1 \\
\hline Pulse2 & “4 44444444444»" & Yes & “10000” & 11 \\
\hline Pulse3 & “2 $222222222222222 » "$ & Yes & “100" & 16 \\
\hline Pulse4 & “ $2 \backslash 1 \checkmark 2121212121212121 \vee "$ & Yes & “10010” & 9 \\
\hline Pulse5 & “1 $111111111111111111 \vee "$ & Yes & “10” & 19 \\
\hline
\end{tabular}

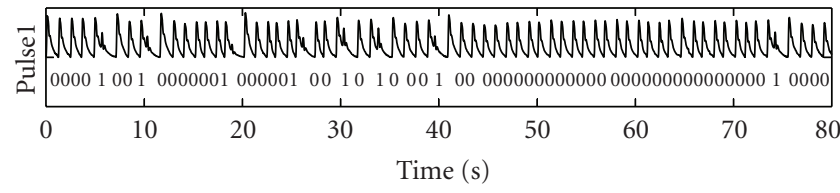

(a)

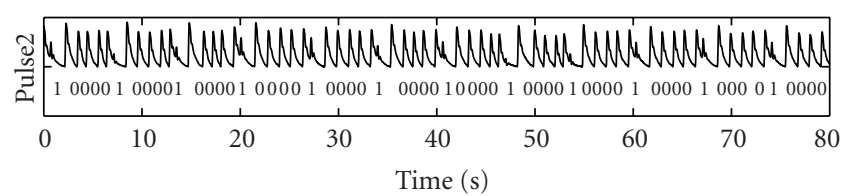

(b)

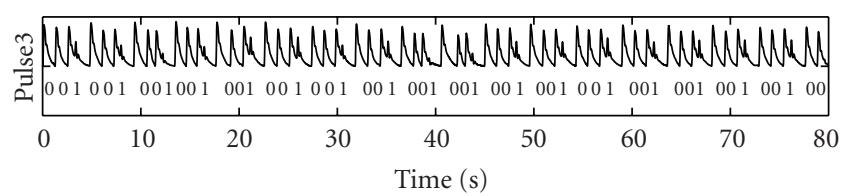

(c)

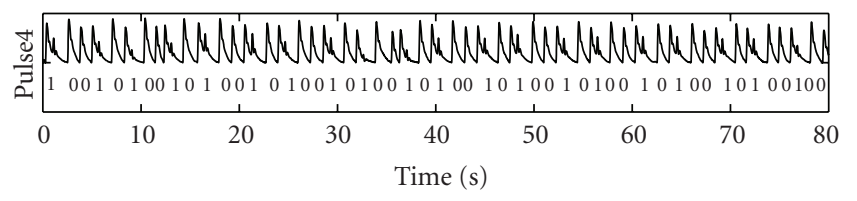

(d)

Figure 11: Five pulses and their SPI sequences.

naïve matching method. We compared 100 SPI sequences, which are periodic or nonperiodic sequences with different length. The naïve matching method requires 1.43 seconds, while our Lempel-Ziv based matching method requires just 1.07 seconds. Furthermore, the longer of the symbolized sequence is, the more time the Lempel-Ziv-based matching method can save.

\section{EXPERIMENTS}

We applied our approach to 140 pulses with different rhythms: swift pulse (20 pulses), rapid pulse (20 pulses), moderate pulse (20 pulses), slow pulse (20 pulses), knotted pulse (20 pulses), running pulse (20 pulses) and intermittent pulse ( 20 pulses). The overall accuracy of the approach is $97.1 \%$. Error arises because the average of PI varies with sex and age. For example, the PI of a healthy female is less than that of healthy male and the PI of a healthy young person is less than that of a healthy old person. In this paper, we do not attempt to account for these influences, but it certainly is the case that the relationship of PI's average to age and sex must be studied in the future research in order to render more accurate classifications. The 20 intermittent pulses in our pulse database exhibit 15 kinds of rhythm variations. Our approach correctly extracts all the MRUs of the 20 intermittent pulses.

In this section, we take five pulses as examples to illustrate the performance of our approach. Figure 11 shows these five pulses and their SPI sequences. Pulse1 is a Knotted Pulse; Pulse2, Pulse3, Pulse4 and Pulse5 are all Intermittent pulses. Their symbolization and subsequences extraction results are as follows:

SPI(Pulse1)

$$
\begin{gathered}
=\text { “0000\#1001\$000000\#100000100 } \\
\text { 1010001\$0000000000000000 } \\
\text { 0000000000000\#1\$0000”; }
\end{gathered}
$$

SPI(Pulse2)

$$
\begin{array}{r}
=\text { “\#1000010000100001000010000100001 } \\
\text { 0000100001000010000100001\$0000"; }
\end{array}
$$

SPI(Pulse3)

$$
\begin{aligned}
=\text { “00\#1001001001001001001001001001001 } \\
\text { 001001001001001001\$00”; }
\end{aligned}
$$

SPI(Pulse4)

$$
\begin{gathered}
=\text { “\#1001010010100101001010010100 } \\
\text { 101001010010100101001\$00”; }
\end{gathered}
$$

SPI(Pulse5)

$$
\begin{gathered}
=\text { “0\#101010101010101010101010 } \\
\text { 101010101010101\$00000000000." }
\end{gathered}
$$

Table 2 lists the Lempel-Ziv analysis results. The subsequence of Pulse1 is nonperiodic and its pulse rate is slow (the average of PIs is 1.25 seconds). We recognize Pulse1 as a knotted pulse. The other four examples, Pulse2, Pulse3, Pulse4, and Pulse5, are all intermittent pulses, each containing different MRUs. 


\section{CONCLUSION}

This paper proposes a Lempel-Ziv-complexity-analysisbased approach to the classification of seven pulse patterns that exhibit different rhythms, and achieves an accuracy of $97.1 \%$. The parameters of VR and VC are first extracted from PI series of pulse waveform, and then are used to judge whether the pulse is arrhythmic or not according to Rule 1. If it is arrhythmic, the PI series should be symbolized and simplified. Combining with Rule 2 and the lemma, the LempelZiv complexity analysis also makes it quite easy to identify the arrhythmic pulse patterns: running pulses, knotted pulses and intermittent pulses. The automatic analysis of pulse rhythms relieves practitioners of the routine work of observing and diagnosing pulse data. Our approach can also be applied to the analysis of the rhythms of other physiological signals.

\section{ACKNOWLEDGMENTS}

This research is supported by National Natural Science Foundation of China (Project 90209020), by the Ph.D. Program Foundation of the Ministry of Education of China (Grant 20040213017), and by the Central/Departmental Fund of The Hong Kong Polytechnic University. We also would like to thank Professors Michael Small and Martin Kyle for their careful proofreading.

\section{REFERENCES}

[1] L. I. Hammer, Chinese Pulse Diagnosis: A Contemporary Approach, Eastland Press, Vista, Calif, USA, 2001.

[2] J. H. Laub, "New non-invasive pulse wave recording instrument for the acupuncture clinic," American Journal of Acupuncture, vol. 11, no. 3, pp. 255-258, 1983.

[3] B. Michael and M. Michael, "Instrument-assisted pulse evaluation in acupuncture," American Journal of Acupuncture, vol. 14, no. 3, pp. 255-259, 1986.

[4] H. Seng, "Objectifying of pulse-taking," Japanese Journal of Oriental Medicine, vol. 27, no. 4, p. 7, 1977.

[5] K.-Q. Wang, L.-S. Xu, Z. Li, D. Zhang, N. Li, and S. Wang, "Approximate entropy based pulse variability analysis," in Proceedings of 16th IEEE Symposium on Computer-Based Medical Systems (CBMS '03), pp. 236-241, New York, NY, USA, June 2003.

[6] W. K. Wang, T. L. Hsu, Y. Chiang, and Y. Y. Lin Wang, "Study on the pulse spectrum change before deep sleep and its possible relation to EEG," Chinese Journal of Medical and Biological Engineering, vol. 12, pp. 107-115, 1992.

[7] L. Y. Wei and P. Chow, "Frequency distribution of human pulse spectra," IEEE Transactions on Biomedical Engineering, vol. 32, no. 3, pp. 245-246, 1985.

[8] H.-L. Lee, S. J. Suzuki, Y. Adachi, and M. Umeno, "Fuzzy theory in traditional Chinese pulse diagnosis," in Proceedings of International Joint Conference on Neural Networks (IJCNN '93), vol. 1, pp. 774-777, Nagoya, Japan, October 1993.

[9] Y.-Z. Yoon, M.-H. Lee, and K.-S. Soh, "Pulse type classification by varying contact pressure," IEEE Engineering in Medicine and Biology Magazine, vol. 19, no. 6, pp. 106-110, 2000.
[10] G. K. Stockman, L. N. Kanal, and M. C. Kyle, "Structural pattern recognition of Carotid pulse waves using a general waveform parsing system," Communications of the ACM, vol. 19, no. 12, pp. 688-695, 1976.

[11] L. Wang, K.-Q. Wang, and L.-S. Xu, "Recognizing wrist pulse waveforms with improved dynamic time warping algorithm," in Proceedings of the 3rd International Conference on Machine Learning and Cybernetics (ICMLC '04), vol. 6, pp. 3644-3649, Shanghai, China, August 2004.

[12] B. H. Wang and J. L. Xiang, "ANN recognition of TCM pulse states," Journal of Northwestern Polytechnic University, vol. 20, no. 3, pp. 454-457, 2002.

[13] S. L. Huang and M. Y. Sun, The Study of Chinese Pulse Image, Chinese People's Sanitation Press, Beijing, China, 1995.

[14] L. S. Zhen, Pulse Diagnosis, Paradigm Publications, Brookline, Mass, USA, 1985.

[15] A. Lempel and J. Ziv, "On the complexity of finite sequences," IEEE Transactions on Information Theory, vol. 22, no. 1, pp. 75-81, 1976.

[16] J. Ziv, "Coding theorems for individual sequences," IEEE Transactions on Information Theory, vol. 24, no. 4, pp. 405412, 1978.

[17] R. Nagarajan, "Quantifying physiological data with LempelZiv complexity-certain issues," IEEE Transactions on Biomedical Engineering, vol. 49, no. 11, pp. 1371-1373, 2002.

[18] L. Y. Huang, Q. X. Sun, and J. Z. Cheng, "Novel method of fast automated discrimination of sleep stages," in Proceedings of the 25th Annual International Conference of the IEEE Engineering in Medicine and Biology Society, vol. 3, pp. 2273-2276, Cancun, Mexico, September 2003.

[19] X.-S. Zhang, R. J. Roy, and E. W. Jensen, "EEG complexity as a measure of depth of anesthesia for patients," IEEE Transactions on Biomedical Engineering, vol. 48, no. 12, pp. 1424-1433, 2001.

[20] S. Mund, "Ziv-Lempel complexity for periodic sequences and its cryptographic application," in Advances in CryptologyEUROCRYPT '91, pp. 114-126, Brighton, UK, April 1991.

[21] K.-Q. Wang, L.-S. Xu, L. Wang, Z. G. Li, and Y. Z. Li, "Pulse baseline wander removal using wavelet approximation," in Proceedings of the 30th Annual Conference of Computers in Cardiology (CinC '03), pp. 605-608, Thessaloniki, Chalkidiki, Greece, September 2003.

[22] M. A. Navakatikyan, C. J. Barrett, G. A. Head, J. H. Ricketts, and S. C. Malpas, "A real-time algorithm for the quantification of blood pressure waveforms," IEEE Transactions on Biomedical Engineering, vol. 49, no. 7, pp. 662-670, 2002.

[23] G. Gratze, J. Fortin, A. Holler, et al., "A software package for non-invasive, real-time beat-to-beat monitoring of stroke volume, blood pressure, total peripheral resistance and for assessment of autonomic function," Computers in Biology and Medicine, vol. 28, no. 2, pp. 121-142, 1998.

[24] K. G. Belani, J. J. Buckley, and M. O. Poliac, "Accuracy of radial artery blood pressure determination with the Vasotrac," Canadian Journal of Anesthesia, vol. 46, no. 5, pp. 488-496, 1999.

[25] L.-S. Xu, D. Zhang, and K.-Q. Wang, "Wavelet-based cascaded adaptive filter for removing baseline drift in pulse waveforms," IEEE Transactions on Biomedical Engineering, vol. 52, no. 11, pp. 1973-1975, 2005.

[26] D. Gusfield and J. Stoye, "Linear time algorithms for finding and representing all the tandem repeats in a string," Journal of Computer and System Sciences, vol. 69, no. 4, pp. 525-546, 2004. 
Lisheng $\mathrm{Xu}$ received B.S. degree in electrical power system automation and M.S. degree in control and automation in mechanical electronics from Harbin Institute of Technology (HIT), China, in 1998 and 2000, respectively. He is currently pursuing the Ph.D. degree at the Biocomputing Research Center of HIT. He is the winner of the $3 \mathrm{rd}$ Best Student Project Proposal selected by IEEE Technical Committee on Computa-

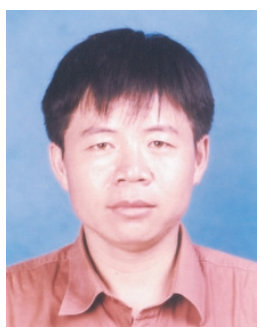
tional Medicine of 2003. He won the Ph.D. Student Award of Excellence of HIT in 2003. He is a Member of Biomedical Engineering Society of Heilongjiang Province. His research interests include medical informatics, nonlinear medical signal processing, pattern recognition, clinical and healthcare information systems. He has published 4 journal papers and 15 conference papers. He participated in several projects on biomedical informatics, human-bodybased diagnosis, and physiological signal detection system.

David Zhang graduated in computer science from Peking University in 1974. He received his M.S. and Ph.D. degrees in computer science from the Harbin Institute of Technology (HIT) in 1982 and 1985, respectively. From 1986 to 1988 , he was a Postdoctoral Fellow at Tsinghua University and then an Associate Professor at the Academia Sinica, Beijing. In 1994, he received his second Ph.D. degree in electrical and computer engineering from the University of Waterloo, Ontario, Canada. Currently, he is a Chair Professor at The Hong Kong Polytechnic University, where he is the Founding Director of the Biometrics Technology Center (UGC/CRC) supported by the Hong Kong SAR Government. He also serves as Adjunct Professor in Tsinghua University, Shanghai Jiao Tong University, Beihang University, Harbin Institute of Technology, and the University of Waterloo. He is the Founder and Editor-in-Chief of International Journal of Image and Graphics (IJIG), Book Editor of Kluwer International Series on Biometrics (KISB), and Program Chair of the International Conference on Biometrics Authentication (ICBA), Associate Editor of more than ten international journals including IEEE Transactions on SMC-A/SMC-C, Pattern Recognition, and is the author of more than 10 books. He is a current Croucher Senior Research Fellow and Distinguished Speaker of IEEE Computer Society.

Kuanquan Wang was born in Sichuan Province, China, in September 1964. He received his B.E. and M.E. degrees in computer science from Harbin Institute of Technology (HIT), China, and his Ph.D. degree in computer science from Chongqing University, in 1985, 1988, and 2001, respectively. From 1988 to 1998 , he worked in the Department of Computer Science, Southwest Normal University, Chongqing city,

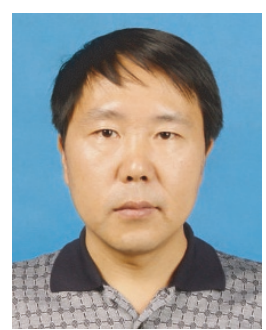
China. From 1998 till now, he has been working in the Biocomputing Research Center of Computer Science and Engineering Department of HIT. Meanwhile, from 2000 to 2001 he was a Visiting Scholar in The Hong Kong Polytechnic University supported by Hong Kong Croucher Funding, and from 2003 to 2004 he was a Research Fellow in the same university. Currently, he is a Professor at the Department of Computer Science and Engineering, and an Associate Director of Biocomputing Research Center in HIT. So far, he has published over 90 papers. His research interests include biometrics, image processing, pattern recognition and biometricsbased diagnosis technology for traditional chinese medicine. He is a Member of the IEEE, an Associate Editor of International Journal of Image and Graphics. Also he is a reviewer of IEEE Transactions on SMC, Pattern Recognition, and so on.

Lu Wang received her B.E. and M.E. degrees in computer science and technology from Harbin Institute of Technology (HIT), Harbin, China, in 2003 and 2005, respectively. She is currently a Ph.D. student at Department of Electrical and Electronic Engineering at the University of Hong Kong. Her research interests include image processing, pattern recognition, signal processing, and virtual reality.

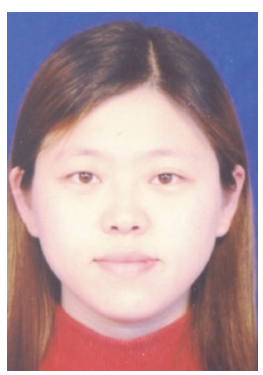

